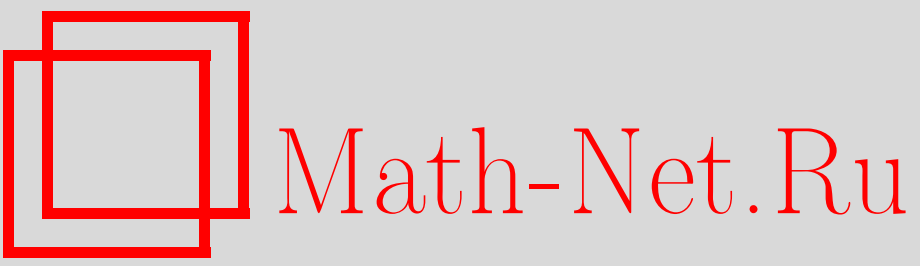

Ю. К. Ачкасов, Н. П. Пильник, Модель общего равновесия с налоговым аудитом и эндогенным выбором между рынком труда и самозанятостью, Матем. моделирование, 2020, том 32, номер 5, 103-125

DOI: https://doi.org/10.20948/mm-2020-05-06

Использование Общероссийского математического портала Math-Net.Ru подразумевает, что вы прочитали и согласны с пользовательским соглашением

http: //www. mathnet.ru/rus/agreement

Параметры загрузки:

IP : 107.22 .136 .117

26 апреля 2023 г., 18:11:54 


\title{
МОДЕЛЬ ОБЩЕГО РАВНОВЕСИЯ С НАЛОГОВЫМ АУДИТОМ И ЭНДОГЕННЫМ ВЫБОРОМ МЕЖДУ РЫНКОМ ТРУДА И САМОЗАНЯТОСТЬЮ
}

\author{
(C) 2020 2. $\quad$ Ю.К. Ачкасов $^{1}$, Н.П. Пильник ${ }^{2,3,4}$
}

${ }^{1}$ Банк России

${ }^{2}$ Национальный исследовательский университет «Высшая школа экономики» ${ }^{3}$ Федеральный исследовательский центр «Информатика и управление» РАН ${ }^{4}$ Научно-исследовательский финансовый институт Минфина РФ u4d@yandex.ru ${ }^{2}$

DOI: $10.20948 / \mathrm{mm}-2020-05-06$

Рассматривается модель общего равновесия, описывающая взаимодействие неоднородных агентов, выбирающих между рынком труда и самозанятостью и государством, играющим в модели роль проверяющего органа (аудитора), контролирующего факт ухода от налогов. Показано, что в случае асимметричности информации увеличивается максимизирующая сумму налоговых поступлений ставка налогообложения. Кривая Лаффера при асимметрии информации приближается к своему аналогу при симметричной информации, если высоки штрафы или бюджет налоговой инспекции. Несмотря на то, что в равновесии сумма выплачиваемых штрафов равна нулю, они оказывают существенное влияние на сумму налоговых поступлений. Являясь эффективным методом устрашения нарушителей налогового законодательства, они увеличивают сумму налоговых сборов с замедляющимся темпом.

Ключевые слова: общее равновесие, самозанятость, налог на прибыль, предложение труда.

\section{GENERAL EQUILIBRIUM MODEL WITH TAX AUDIT} AND ENDOGENOUS CHOICE BETWEEN LABOUR MARKET AND SELF-EMPLOYMENT

\author{
Yu.K. Achkasov ${ }^{1}$, N.P. Pilnik ${ }^{2,3,4}$ \\ ${ }^{1}$ Bank of Russia \\ ${ }^{2}$ National Research University Higher School of Economics \\ ${ }^{3}$ Federal Research Center «Computer Science and Control» of the RAS \\ ${ }^{4}$ Financial Research Institute of the Ministry of finance of the Russian Federation
}


The article discusses a general equilibrium model that describes the interaction of heterogeneous agents, who choose between the labor market and self-employment, and the state, which plays the role of an inspection body (auditor) in the model, which controls the fact of tax evasion. It is shown that in the case of asymmetric information, the tax rate that maximizes the amount of tax revenues increases. With the asymmetry of information, the Laffer curve approaches its counterpart with symmetric information if fines or the budget of the tax inspectorate are high. Despite the fact that in balance the amount of fines paid is zero, they have a significant impact on the amount of tax revenues. Being an efficient way of deterring tax violators, they increase the amount of tax collections at a slowing rate.

Keywords: general equilibrium, self-employment, income tax, labour supply.

\section{1. Введение}

Уход от налогов является серьезной проблемой для государственных финансов в современном мире. Как утверждают в [1], развивающиеся страны страдают от ухода от налогов даже больше, чем развитые, так как механизмы контроля за выполнением налоговых обязательств в развивающихся странах гораздо менее эффективны. В [2] подчеркивается, что важными факторами, с точки зрения собираемости налогов, является институциональная среда и возможности государственных органов по контролю за собираемостью.

Выбор фирм, в каком секторе экономики вести свою деятельность (формальном или неформальном), определяется системой стимулов и имеющих место в экономике угроз, в том числе со стороны органов государственного контроля. С одной стороны, пребывание в теневом секторе экономики дает фирмам возможность уклониться от некоторых налогов. С другой стороны, могут существовать преимущества и у легального сектора экономики с точки зрения фирм. Например, в [3] предполагается, что неформальные фирмы понесут дополнительные издержки. Например, если они занимаются импортом сырья и материалов, то не смогут получить налоговые вычеты. Как правило, НДС по импортному сырью возмещается, если это сырье используется в дальнейшем производстве. И обычно достаточно трудно избежать уплаты налогов на таможне, так что даже предприятия, работающие в теневом секторе, должны будут оплатить эти налоги.

Другая идея, объясняющая преимущества формального сектора экономики, предложена в [1]. Авторы предполагают, что фирмы могут пользоваться услугами банковских посредников только находясь в формальном секторе экономики или они могут уйти в теневой сектор, в котором использование банковских услуг невозможно. Банки помогают легальным фирмам 
инвестировать и защищают их денежные средства от инфляции, в то время как нелегальные фирмы страдают и от инфляции, и от недостатка инвестиций. Эта модель базируется на предпосылке, что неформальный сектор экономики использует наличные платежи, что является весьма распространенным явлением в развивающихся экономиках. Например, в [4] обсуждаются различные способы использования «черного нала» российскими фирмами в 1990-е годы.

В ситуации, когда нелегальный статус фирмы имеет и свои преимущества, и свои недостатки, у государства появляется возможность построить оптимальную налоговую систему, использующую плюсы и минусы теневого сектора экономики таким образом, чтобы суммарные налоговые доходы были максимальны. С другой стороны, если у ухода от налогов нет однозначных минусов с точки зрения фирмы, а налоговые ставки являются экзогенными, регулятору придется заставлять фирмы платить налоги путем проведения проверок и штрафования тех, кто пытался скрыть свои истинные доходы.

В данной работе используется именно этот подход при описании взаимодействия между налогоплательщиками и государством. Во втором разделе мы кратко опишем существующие в литературе теоретические модели налогового аудита. Третий раздел данной работы описывает агентов и равновесие в модели с эндогенным выбором между работой на рынке труда и самозанятостью. В четвертом разделе анализируется ситуация с асимметрией информации, описана задача аудитора и представлен оптимальный механизм, обеспечивающий сбор налогов. В этом же разделе приведен краткий обзор используемых математических и теоретико-игровых моделей, применяемых в данной работе для анализа и решения задачи аудитора. В конце раздела проведен анализ оптимального решения задачи аудитора и его максимально возможного выигрыша. В пятом разделе полученные теоретические выводы проиллюстрированы при помощи численных расчетов. Наконец, в шестом разделе обсуждаются полученные результаты и дальнейшие направления исследования.

\section{2. Обзор литературы}

Одной из первых работ, посвященной моделированию аудита собираемости налогов, является [5]. Авторы предположили, что у различающихся по склонности к риску агентов есть выбор между безрисковой и рисковой занятостью. Выбрав безрисковую занятость, агент наверняка получал фиксированный доход $c$. Рисковая занятость может принести агенту доход $a$ или $b$ с разными вероятностями. Из-за того что доход агента случаен и не 
наблюдаем налоговым органом, у агента есть стимул и возможность уклоняться от части налогов. В этих условиях, варьируя налоговую ставку и вероятность налоговой проверки, налоговые власти могут влиять на величину собранных налогов или на общественное благосостояние.

В модели [6] также предполагается, что доход агентов может быть случаен, при этом его распределение не дискретно, как в работе [5], а непрерывно. Никто, кроме самого агента, не знает величины его дохода $y$. Аудитор знает исключительно распределение $y$ между агентами и доход $x$, который (не всегда честно) сообщается каждым агентом аудитору. Авторы находят оптимальный механизм, который максимизирует налоговые доходы государства. Таким оптимальным механизмом аудита оказывается проверка агентов, которые говорят, что они получают низкие доходы, с очень высокой вероятностью. Остальных агентов аудитор проверять не будет вообще, чтобы не тратить напрасно ограниченный бюджет.

В [7] предполагают, что доход экономических агентов $y$ не задается экзогенно, а формируется благодаря производству товара или услуги. Авторы предложили, что каждый агент имеет свой тип $z$, который увеличивает выпуск в $z$ раз. В статье исследуется, каким образом аудитор может максимизировать сумму налоговых поступлений, если он будет использовать только информацию о заявленном агентами доходе, только информацию об объемах спроса на труд со стороны производителей или информацию об обеих этих величинах.

В случае когда аудитор использует только информацию о заявленном доходе, результат [7] отличается от результатов [6] несущественно, несмотря на введенное в модель производство. Если налоговому инспектору доступна информация лишь о спросе на труд со стороны производителей (но не о доходе), то он выберет другую стратегию аудита: проверять фирмы с высоким спросом на труд и не проверять фирмы, спрос на труд у которых низок. С одной стороны, этот вывод сильно отличается от предыдущего вывода авторов, когда аудитор имеет информацию о заявленных агентами доходах. Разница состоит в том, что в случае с известными декларируемыми доходами аудитор проверял наименее производительные фирмы, в случае с наблюдаемым спросом на труд проверяются, напротив, наиболее производительные фирмы. С другой стороны, такая ситуация не противоречит экономическим реалиям: зачастую налоговая инспекция проверяет крупные (в терминах занятости) фирмы, оставляя маленькие предприятия без внимания.

Третья модель, когда аудитор использует информацию и о спросе на труд, и о декларируемом доходе, в общем случае не имеет математического решения. Тем не менее авторы попытались рассмотреть некоторые частные 
случаи в рамках этой модели и изучить примерные взаимосвязи между стратегиями игроков. В итоге авторы приходят к не очень строгому, но непротиворечивому выводу, объединяющему выводы предыдущих двух моделей: аудитор будет очень строг к крупным фирмам, которые декларируют низкий доход. Напротив, «расслабиться» смогут маленькие игроки, декларирующие большую прибыль. Крупные фирмы, декларирующие большую прибыль или маленькие фирмы, декларирующие маленькую прибыль, как раз будут находиться на границе между теми, кого аудитор проверяет особенно тщательно, и теми, кого он не проверяет вообще.

Далее мы ограничим наше изучение оптимальных стратегий налогового надзора случаем, когда агенты сообщают (необязательно правдиво) только свой доход. Однако, в отличие от [7], мы не будем предполагать, что все агенты становятся производителями. В нашей модели некоторые из агентов выбирают статус наемных работников, а другие - статус предпринимателей. Такая модификация введет рынок труда в экономику, а зарплата перестанет быть экзогенной, а будет определяться взаимодействием спроса на труд со стороны фирм и предложением труда со стороны рабочих.

Рассматриваемая модель будет принадлежать к классу моделей общего равновесия с неоднородными агентами, предложенных в [8] и описанных, в частности, в [9]. Данную концепцию мы будем использовать как общую рамку моделирования, наполняя ее описанием интересующих нас экономических механизмов и взаимодействий. Описание способностей агентов к предпринимательской деятельности мы формулируем так же, как это было сделано в [7]. В рамках моделей общего равновесия похожим образом предпринимательскую способность описывали в [10-12]. В [13,14] используется модель общего равновесия для изучения влияния налоговой политики на основные экономические переменные. Однако в указанной работе предполагается, что информация симметрична и агенты платят налоги полностью. В данном исследовании будет изучено взаимодействие между налогоплательщиками и налоговой инспекцией, которая будет разрабатывать механизм налоговых проверок для обеспечения максимальной собираемости налогов.

\section{3. Модель экономики}

3.1. Агенты. Рассмотрим континуум экономических агентов, каждый из которых описывается параметром $z$, непрерывно распределенным на промежутке $Z \equiv[\underline{z}, \bar{z}](\underline{z}>0)$ в соответствии с функцией распределения $G(z)$ и функцией плотности вероятности случайной величины $g(z)>0$.

Каждый агент обладает одной единицей труда, является нейтральным к риску и безразличным к досугу индивидом. Агент может пойти работать 
наемным рабочим на совершенно конкурентный рынок труда и получать при этом равновесную заработную плату $w$ или открыть свое собственное предприятие (которому он посвятит все свое время).

Каждый предприниматель ${ }^{1}$ имеет производственную функцию $y=z f(l)$. Параметр $z$ может, таким образом, интерпретироваться как «управленческая способность», как это было сделано ранее в статье [7]. Мы предполагаем, что функция $f(\cdot)$ обладает «стандартными» свойствами производственной функции: $f(0)=0, f(\cdot) \in \mathbf{C}^{2}\left(R_{++}^{2}\right)$ с $f^{\prime}>0$ и $f^{\prime \prime}<0$. Для простоты мы также предполагаем выполнение условий Инады: $\lim _{x \rightarrow 0} f^{\prime}(x)=\infty, \lim _{x \rightarrow+\infty} f^{\prime}(x)=0$.

Предприниматель выбирает оптимальный объем нанимаемого труда $l^{*}$, который максимизирует его прибыль $z f(l)-w l$. Осуществив оптимальный выбор, агент получит прибыль $\pi(z, w)$. С учетом того, что полезность зависит только от получаемого дохода, агент станет самозанятым, если

$$
\pi(z, w) \geqslant w \text {. }
$$

3.2. Равновесие без налогов. Равновесие в такой экономике может быть качественно описано следующим образом: некоторые агенты, для которых (1) не выполняется, будут работать на рынке труда, предлагая по одной единице труда каждый, а другие, для которых (1) выполняется, будут нанимать количество труда $l^{*}$, максимизирующее их прибыль.

Определим $z_{0}$ как решение $\pi\left(z_{0}, w\right)=w$ для заданного уровня зарплаты. В [13] показано, что агенты, имеющие тип $z$, меньший, чем $z_{0}$, будут работать на рынке труда, предлагая одну единицу труда. Остальные будут нанимать $l^{*}(z, w)$ работников.

Таким образом, равновесная заработная плата будет определяться взаимодействием рыночного предложения труда и рыночного спроса на труд:

$$
G\left(z_{0}\right)=\int_{z_{0}}^{\bar{z}} l^{*}(z, w) d G(z)
$$

3.3. Равновесие с налогами на прибыль. Предположим, что вводится пропорциональный налог на прибыль с постоянной экзогенной ставкой $\tau \in(0,1)$. Тогда условие $(1)$, при котором агент становится самозанятым, изменится:

\footnotetext{
${ }^{1}$ Или самозанятый, в дальнейшем по ходу работы мы будем рассматривать эти понятия как синонимы. О различии между этими понятиями см. [13].
} 
Модель общего равновесия с налоговым аудитом и эндогенным выбором ...

$$
(1-\tau) \pi(z, w) \geqslant w .
$$

Это повысит минимальный уровень управленческих способностей $z$, необходимый для того, чтобы стать самозанятым. Теперь ими станут только агенты с $z \geqslant z_{\tau}>z_{0}$ (где $z_{\tau}$ является решением $\left.(1-\tau) \pi\left(z_{\tau}, w\right)=w\right)$.

Равновесие на рынке труда модифицируется:

$$
G\left(z_{\tau}\right)=\int_{z_{\tau}}^{\bar{z}} l^{*}(z, w) d G(z)
$$

\section{4. Аудит}

Предположим теперь, что параметр $z$ не наблюдается никем, кроме самого агента. Тогда государственные органы столкнутся с проблемой «неблагоприятного отбора»: самозанятые сделают все возможное, чтобы избежать уплаты налогов, а правительство должно будет создать такой механизм принуждения, который заставит агентов платить налоги.

4.1. Описание модели. Описание механизма аудита в нашей модели будет основываться на конструкции, предложенной в [7]. Предполагается, что агентство, ответственное за сбор налогов (налоговая инспекция) является принципалом. Цель агентства - собрать максимальное количество налогов. Правительство экзогенно выбирает налоговую ставку $\tau$ и бюджет налоговой инспекции $C$, который используется ей для осуществления своих функций.

Функционирующие в экономике фирмы должны платить налог на прибыль в размере $\tau \pi(z, w)$, но $z$ является ненаблюдаемой величиной для налоговой инспекции, которая, однако, знает распределение этой величины по всей генеральной совокупности агентов. Мы также предположим, что принципал не может знать прибыль фирм и величину их спроса на труд. Фирмы сообщают в налоговую инспекцию $x$ (декларируемую прибыль, которая, возможно, отличается от их истинной прибыли) и платят $\tau x$ в качестве налога.

Налоговая инспекция может проводить проверку агентов после того, как они сообщили ей $x$. Если инспекция проверяет агента, то вся его частная информация (его тип $z$, истинная величина прибыли и его спрос на труд) раскрывается. Если $x$ оказывается меньше, чем истинная прибыль, фирма компенсирует государству ущерб и платит штраф, пропорциональный величине скрытой прибыли (используется постоянный коэффициент $\mu>1$ ). Таким образом, после проверки фирма должна будет заплатить

$$
\begin{cases}\mu \tau(\pi(z, w)-x), & \text { если } x<\pi(z, w) \\ 0, & \text { если } x \geq \pi(z, w) .\end{cases}
$$


Мы предполагаем, что аудитор может различать работников и фирмы без всяких издержек.

Игра между принципалом и агентами развивается по следующему сценарию. Сначала природа сообщает агентам их тип $z$ в соответствии с известным всем распределением $G(z)$. После этого на первом ходу игры инспектор выбирает долю агентов, для которых будет проведен аудит $p$. На втором шаге агенты, зная свой тип и $p$, решают, будут ли они работать на рынке труда или станут самозанятыми. Если они выберут последний вариант, они также принимают решение о количестве нанятых сотрудников $l$ и о величине декларируемой прибыли $x$.

Проведение налоговых проверок в модели является затратным мероприятием. Налоговая инспекция несет постоянные положительные предельные издержки аудита в размере $c$. Общие издержки проверки одного агента с вероятностью $p$ составят $c p$. Мы предполагаем, что бюджета налоговой инспекции $C$ недостаточно, чтобы проводить проверки с такой интенсивностью, чтобы заставить все фирмы предоставлять правдивую информацию и, следовательно, платить все налоги.

4.2. Рациональные стратегии фирм. На втором шаге игры, зная стратегию аудитора, агенты будут максимизировать свой ожидаемый доход. Вопервых, так как спрос фирм на труд не наблюдается налоговой инспекцией, фирма всегда будет выбирать $l^{*}(z, w)$, который принесет ей валовую прибыль в размере $\pi(z, w)$. Во-вторых, агент никогда не выберет декларируемую прибыль $x$, большую, чем величина его истинной прибыли. В-третьих, если $p \geqslant 1 / \mu$, фирма всегда будет декларировать свою истинную прибыль.

$$
\text { def }
$$

Это можно показать, сравнив ожидаемую прибыль $\Pi=\pi(z, w)-x \tau-$ $-\mu p \tau(\pi(z, w)-x)$ с чистой прибылью $(1-\tau) \pi(z, w)$. Чистая прибыль будет меньше ожидаемой, если $(\pi-x)(1-\mu p)>0$, что невозможно, если одновременно выполняется $\pi>x$ и $p>1 / \mu$.

Напротив, можно показать, что при $p<1 / \mu$ фирма будет всегда декларировать меньшую прибыль по сравнению с той, что она зарабатывает на самом деле, потому что $\Pi>(1-\tau) \pi$.

Аудитор никогда не будет проверять агентов с вероятностью, большей, чем $1 / \mu$. Если инспектор все же будет проверять хотя бы одного агента с большей вероятностью, то он сможет уменьшить эту вероятность до уровня $1 / \mu$, не уменьшив поступлений от этого агента. Высвободившиеся средства 
аудитор сможет потратить на аудит других агентов, что увеличит налоговые поступления.

\section{3. Принцип раскрытия информации и ограничения совместимо-} сти по стимулам. В [16] было показано, что процесс переговоров с асимметричной информацией может быть представлен как игра с прямым раскрытием информации. В течение нашего анализа мы неоднократно будем пользоваться принципом раскрытия информации. Основная идея решения модели с игроком, тип которого неизвестен, показана в [17].

В литературе существует большое количество моделей с задачей аудитора. [15] предлагает строгое математическое решение похожей задачи, которая возникает в контексте отношения между собственником фирмы и наемным менеджером. В [6] применяют принцип решения моделей такого типа к проблемам ухода от налогов. В [7] совершенствуют модель [6], добавляя производителей в экономику, а мы рассмотрим эту модель в предположении эндогенного равновесия на рынке труда.

Интуиция принципа раскрытия информации заключается в следующем. Агент сообщает свой «тип» $z^{\prime}$ аудитору. Важно отметить, что озвученный тип $z^{\prime}$ может отличаться от истинного типа $z$. Далее аудитор и агент выбирают свои стратегии так, как будто бы истинным типом фирмы являлся $z^{\prime}$, то есть набор $\left(p\left(z^{\prime}\right), x\left(z^{\prime}\right)\right)$. Конечно, налоговая инспекция сделает все возможное, чтобы заставить агентов раскрыть свой тип честно. Это даст нам ограничения совместимости по стимулам ${ }^{2}$.

Смысл этих ограничений прост: каждый агент $z$ должен достигать большего уровня благосостояния, если он сообщит $z$, а не $z^{\prime}$ (для любых возможных $\left.z^{\prime} \neq z\right)$.

$$
\begin{aligned}
& \Pi\left(z, z^{\prime}, w\right) \stackrel{d e f}{=} \pi(z, w)-x\left(z^{\prime}\right) \tau-\mu p\left(z^{\prime}\right) \tau\left(\pi(z, w)-x\left(z^{\prime}\right)\right) \leqslant \\
& \leqslant \pi(z, w)-x(z) \tau-\mu p(z) \tau(\pi(z, w)-x(z)) \forall z, z^{\prime},
\end{aligned}
$$

что упрощается до

$$
x(z) \tau+\mu p(z) \tau(\pi(z, w)-x(z)) \leqslant x\left(z^{\prime}\right) \tau+\mu p\left(z^{\prime}\right) \tau\left(\pi(z, w)-x\left(z^{\prime}\right)\right) \stackrel{d e f}{=} U\left(z, z^{\prime}, w\right) .
$$

В данном случае $U\left(z, z^{\prime}, w\right)$ следует трактовать как общий объем поступлений в бюджет (налогов и штрафов), собранных с агента с типом $z$, который заявил тип $z^{\prime}$.

\footnotetext{
${ }^{2}$ В дальнейшем мы будем сокращать это название до IC от английского «incentive compatibility constraints».
} 
Условие (5) означает, что $z=\arg \min _{z^{\prime}} U\left(z, z^{\prime}, w\right)$. Необходимое условие этого $-\left.\frac{\partial U\left(z, z^{\prime}, w\right)}{\partial z^{\prime}}\right|_{z^{\prime}=z}=0$. Тогда, определяя $U(z, w) \stackrel{d e f}{=} U(z, z, w)$, мы получим

$$
U^{\prime}(z, w) \stackrel{\text { def }}{=} \frac{\partial U(z, w)}{\partial z}=\frac{\partial U(z, z, w)}{\partial z}=\mu p(z) \tau f\left(l^{*}(z, w)\right) .
$$

Используем (5) дважды, чтобы показать, что $p(z)$ должно быть неубывающей функцией (это будет достаточным условием (5)), чтобы удовлетворять IC:

$$
U(z, z, w) \leqslant U\left(z, z^{\prime}, w\right), U\left(z^{\prime}, z^{\prime}, w\right) \leqslant U\left(z^{\prime}, z, w\right) .
$$

Просуммировав два неравенства и приведя подобные члены, получим

$$
\left(\pi(z, w)-\pi\left(z^{\prime}, w\right)\right)\left(p(z)-p\left(z^{\prime}\right)\right) \leqslant 0 .
$$

Взяв $z>z^{\prime}$ без потери общности, мы получаем, что первая скобка положительна, так как прибыль возрастает по $z$. Тогда $p(z) \leqslant p\left(z^{\prime}\right)^{3}$. Качественно это можно объяснить тем, что если бы $p(z)$ убывало, то агенту было бы выгодно притворяться агентом с меньшим уровнем $z-$ и именно этого не хочет допустить налоговый инспектор. С неубывающей $p(z)$ стимул агента с более высоким уровнем $z$ притвориться агентом с более низким уровнем $z$ и платить меньше налогов компенсируется увеличившейся частотой налоговых проверок.

4.4. Множество потенциальных предпринимателей. Так как аудитор может различать работников и предпринимателей, важно определить подмножество $Z_{e} \subseteq Z$ типов таких, что агенты с этими типами могут захотеть стать предпринимателями (при какой-то стратегии инспектора). Важно понимать, что, возможно, в равновесии окажется меньше предпринимателей, нежели предполагает множество $Z_{e}$, которое всего лишь определяет типы, на множестве которых налоговый инспектор будет строить свои стратегии.

Отметим, во-первых, что $\left[z_{\tau}, \bar{z}\right] \subseteq Z_{e}$. Все агентами с типами $z \in\left[z_{\tau}, \bar{z}\right]$ будут самозанятыми, даже если они будут платить все налоги, в соответствии с (3). Как было показано в подразделе 4.2 , для $p \leqslant 1 / \mu$ выполняется $\Pi \geqslant(1-\tau) \pi$. Это приведет к тому, что для $z \in\left[z_{\tau}, \bar{z}\right] \Pi \geqslant w$, что означает, что эти агенты будут предпринимателями для любой стратегии налоговой инспекции $p \leqslant 1 / \mu$.

${ }^{3}$ В [17] предлагается похожее доказательство. 
Напротив, можно сказать, что для $\forall z \in Z_{e} z \geqslant z_{0}$. В соответствии с (1), все агенты с $z<z_{0}$ будут получать зарплату, которая будет выше, чем прибыль до уплаты налогов. В соответствии с определением ожидаемой прибыли П, она всегда не больше, чем $\pi$. Таким образом, агенты с $z<z_{0}$ будут всегда иметь $\Pi<w$.

Очень важным предположением является то, что налоговая инспекция может различать фирм и работников. В соответствии с ним, ни одна фирма с типом $z>z_{0}$ не будет притворяться агентом с типом $z<z_{0}$. Если бы какаято фирма делала это, налоговая инспекция сразу бы поняла, что фирма лжет, так как все агенты такого типа находятся на рынке труда. Это эквивалентно бесплатному аудиту с вероятностью, равной единице, который однозначно сделает бессмысленным для всех фирм сообщать тип $z<z_{0}$. Этот же аргумент будет использоваться, чтобы запретить агентам притворяться кем-либо не из множества $Z_{e}$ : аудитор не поверит ни одному сигналу, означающему, что какой-то самозанятый обладает типом $z \in Z \backslash Z_{e}$.

Мы предлагаем следующий способ построить множество $Z_{e}: Z_{e}=$ $=\left[z^{*}, \bar{z}\right] \mathrm{c} z^{*}$ таким, что:

$$
\begin{aligned}
& \Pi\left(z^{*}, z^{*}, w\right) \geqslant w ; \\
& \forall z<z^{*} \Pi\left(z, z^{*}, w\right)<w .
\end{aligned}
$$

Качественно это означает, что агент $z^{*}$ может иметь прибыль, не меньшую, чем зарплата. Все агенты с $z<z^{*}$ не имеют права создать предприятие, если они будут сообщать свой истинный тип $z$. Однако у них есть возможность стать предпринимателями, но для этого им придется сообщить тип $z^{*}$. Последнее неравенство означает, что у них не будет стимулов поступать таким образом и они останутся на рынке труда. Как уже обсуждалось выше, $z^{*}$ должно принадлежать отрезку $\left[z_{0}, z_{t}\right]$.

Можно упростить это определение. Во-первых, заметим, что П $\left(z, z^{*}, w\right)$ строго возрастает по «настоящему» типу $z$. Несложные расчеты и лемма Хоттелинга дают

$$
\partial \Pi\left(z, z^{*}, w\right) / \partial z=\left(1-\mu p\left(z^{*}\right) \tau\right) f\left(l^{*}(z, w)\right) .
$$

Так как в силу условий Иннады $l^{*}(z, w)>0$ и так как $\mu p \leqslant 1$ и $\tau<1$, мы имеем

$$
\partial \Pi\left(z, z^{*}, w\right) / \partial z=\left(1-\mu p\left(z^{*}\right) \tau\right) f\left(l^{*}(z, w)\right)>0 .
$$


Так как функция $\Pi\left(z, z^{*}, w\right)$ является непрерывной и строго возрастающей по $z$, а условия (8) и (9) выполняются, это значит, что $\lim _{z \rightarrow z^{*}-0} \Pi\left(z, z^{*}, w\right)=w$, что, в свою очередь, вместе с (8) даст нам П $\left(z^{*}, z^{*}, w\right)=w$. При этом условие (9) автоматически выполняется. Таким образом, новое, более простое определение $Z_{e}$ будет выглядеть следующим образом:

$$
Z_{e}=\left[z^{*}, \bar{z}\right] \text {, где } z^{*} \text { такое, что } \Pi\left(z^{*}, z^{*}, w\right)=w \text {. }
$$

def

Можно вывести, что функция $\Pi(z, w)=\Pi(z, z, w)$ строго возрастает по $z$ для $z \in Z_{e}{ }^{4}$.

Возьмем $z_{a}<z_{b}$. В соответствии с (11), П $\left(z_{a}, z_{a}, w\right)<\Pi\left(z_{b}, z_{a}, w\right)$. Согласно IC (8), П $\left(z_{b}, z_{a}, w\right) \leqslant \Pi\left(z_{b}, z_{b}, w\right)$. Поэтому

$$
\Pi\left(z_{a}, w\right)=\Pi\left(z_{a}, z_{a}, w\right)<\Pi\left(z_{b}, z_{b}, w\right)=\Pi\left(z_{b}, w\right) .
$$

Из этого свойства следует, что $\forall z>z^{*} \Pi(z, w)>w$. Это значит, что все агенты с $z \geqslant z^{*}$ станут предпринимателями.

Также становится ясно, что если $z^{*}$ существует, тогда множество $Z_{e}$, определенное в (11), становится множеством действующих предпринимателей, а не только потенциальных. Это так, потому что все агенты с $z \geq z^{*}$ станут бизнесменами, а все агенты с $z<z^{*}$ пойдут работать на рынок труда. Теперь равновесие на рынке труда будет определяться похожим на предыдущие случаи (без асимметрии информации) образом:

$$
G\left(z^{*}\right)=\int_{z}^{\bar{z}} l^{*}(z, w) d G(z)
$$

Рис.1 показывает идею построения «множества потенциальных предпринимателей»: агенты с предпринимательскими способностями $z<z_{0}$ остаются работниками, агенты с $z \geq z_{\tau}$ однозначно будут самозанятыми. Кроме того, предпринимательской деятельностью могут также заниматься агенты с уровнем способностей $z \in\left[z^{*}, z_{\tau}\right]$, при этом они не смогут полностью платить налоги. Те агенты, которые обладают способностями $z \in\left(z_{0}, z^{*}\right)$, могут стать самозанятыми, однако для этого им придется завысить свой «раскрываемый» тип $z^{\prime}$ до уровня $z^{*}$.

\footnotetext{
${ }^{4}$ Очевидно, что функция также строго убывает по $w$.
} 
Модель общего равновесия с налоговым аудитом и эндогенным выбором ...

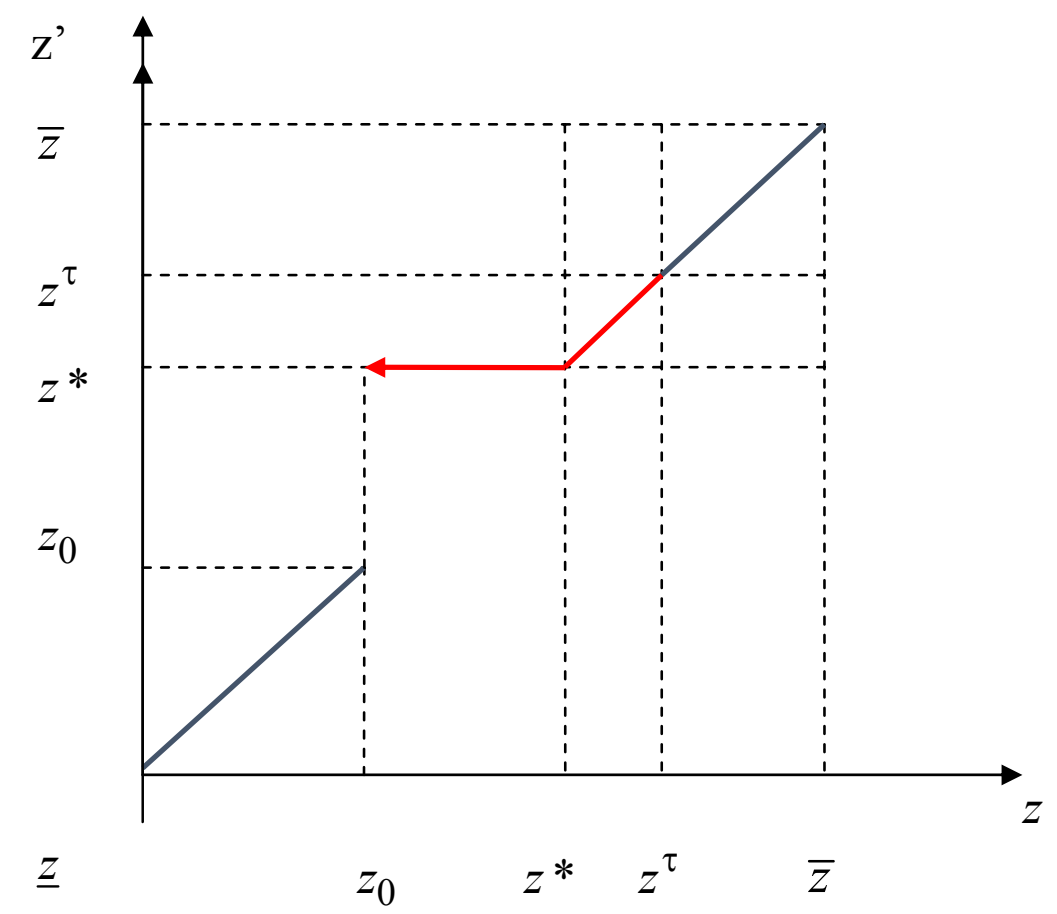

Рис.1. Множество потенциальных предпринимателей.

4.5. Задача аудитора. Налоговая инспекция хочет собрать максимальное количество налогов, разрабатывая механизм $(p(z), x(z))^{5}$ при выполнении следующих ограничений:

- Ограничения допустимости, накладываемые на функции $p(z), x(z)$ : $p(z) \in[0,1 / \mu]$ и $x(z) \in[0, \pi(z, w)]$.

- Условия совместимости по стимулам в форме (6) и достаточное условие (7) $(p(z)$ не возрастает по $z)$.

- Бюджетное ограничение аудитора $\int_{z^{*}}^{\bar{z}} c p(z) d G(z) \leq C$, причем $C$ недостаточно высокое ${ }^{6}$ для того, чтобы проверять всех с вероятностью $1 / \mu$.

- Определение $z^{*}$, данное нами в (11).

- Условия равновесия на рынке труда (13).

Задача налоговой инспекции выглядит следующим образом:

\footnotetext{
${ }_{5}^{5}$ Мы предполагаем, что $p(z), x(z)$ - кусочно-непрерывные функции.

${ }^{6}$ Точнее сказать, $C<\frac{1}{\mu} \int_{Z_{e}} c d G(z)$. В случае когда это условие не выполняется, решение аудитора становится тривиальным: он проверяет всех с вероятностью $1 / \mu$, и государство получает всю сумму налогов, которую оно получало при полной симметрии информации.
} 


$$
\left\{\begin{array}{l}
\int_{z}^{\bar{z}} U(z, w) g(z) d z \rightarrow \max _{(p(z), x(z)), z^{*}} \\
\text { s.t.: } \\
p(z) \in[0,1 / \mu] \\
x(z) \in[0, \pi(z, w)] \\
z^{*} \in\left[z_{0}, z_{\tau}\right] \\
U^{\prime}(z, w)=\mu p(z) \tau f\left(l^{*}(z, w)\right) \\
p(z) \text { не возрастает } \\
\int_{z^{*}}^{\bar{z}} c p(z) d G(z) \leq C \\
\Pi\left(z^{*}, z^{*}, w\right)=w \\
G\left(z^{*}\right)=\int_{z^{*}}^{\bar{z}} l^{*}(z, w) d G(z)
\end{array}\right.
$$

Возможно проинтегрировать целевую функцию по частям:

$$
\begin{aligned}
& \int_{z}^{\bar{z}}{ }^{*} U(z, w) g(z) d z=\left.U(z, w) G(z)\right|_{z} ^{\bar{z}} \bar{z}^{*}-\int_{z}{ }^{*} U^{\prime}(z, w) G(z) d z= \\
& =U(\bar{z}, w)-G\left(z^{*}\right) U\left(z^{*}, w\right)-\int_{z^{*}}^{\bar{z}} U^{\prime}(z, w) G(z) d z= \\
& =U(\bar{z}, w)+\int_{z}^{\bar{z}} U^{\prime}(z, w) d z-G\left(z^{*}\right) U\left(z^{*}, w\right)-\int_{z}^{\bar{z}} U^{\prime}(z, w) G(z) d z= \\
& =U(\bar{z}, w)-G\left(z^{*}\right) U\left(z^{*}, w\right)+\int_{z}^{\bar{z}} U^{\prime}(z, w)(1-G(z)) d z .
\end{aligned}
$$

Заметим, что $\Pi\left(z^{*}, z^{*}, w\right)=\pi\left(z^{*}, w\right)-U\left(z^{*}, w\right)$ по определению. Тогда ограничение (11) даст нам $U\left(z^{*}, w\right)=\pi\left(z^{*}, w\right)-w$. Произведя несколько замен, можно упростить целевую функцию до вида

$$
\left(\pi\left(z^{*}, w\right)-w\right)\left(1-\int_{z}^{\bar{z}} l^{*}(z, w) g(z) d z\right)+\int_{z}^{\bar{z}} \mu p(z) \tau f\left(l^{*}(z, w)\right) \gamma(z) g(z) d z,
$$

где $\gamma(z)=(1-G(z)) / g(z)$ - отношение Миллса (или обратная мера риска).

Решение задачи аудитора. Поиск $p(z)$. Важно заметить, что бюджетное ограничение для налоговой инспекции будет выполняться как равенство. Если бы оно не выполнялось как равенство, налоговый инспектор смог бы всегда увеличить значение целевой функции, не нарушая этим бюджетного ограничения, проверив еще одного агента с вероятностью $1 / \mu$ и заставив его тем самым заплатить налог полностью. Теперь ненадолго забудем 
про достаточное условие про невозрастание функции $p(z)$ и построим функцию Лагранжа, используя бюджетное ограничение аудитора.

$$
\begin{aligned}
& \mathfrak{L}=\left(\pi\left(z^{*}, w\right)-w\right)\left(1-\int_{z^{*}}^{\bar{z}} l^{*}(z, w) d G(z)\right)+\int_{z^{*}}^{\bar{z}} \mu p(z) \tau f\left(l^{*}(z, w)\right) \gamma(z) g(z) d z- \\
& -\lambda\left(\int_{z}^{\bar{z}} c p(z) d G(z)-C\right)=\left(\pi\left(z^{*}, w\right)-w\right)\left(1-\int_{z}^{\bar{z}} l^{*}(z, w) d G(z)\right)+ \\
& +\int_{z^{*}}^{\bar{z}} p(z)\left[\mu \tau f\left(l^{*}(z, w)\right) \gamma(z)-\lambda c\right] g(z) d z+\lambda C .
\end{aligned}
$$

Для любого $z^{*} \in\left[z_{0}, z_{\tau}\right]$ лагранжиан зависит линейно от $p(z)$. В данном месте нам придется ввести достаточно сильную предпосылку, как это было сделано в [7]:

$$
f\left(l^{*}(z, w)\right) \gamma(z) \text { строго убывает по } z \text {. }
$$

Тогда функция $\mu \tau f\left(l^{*}(z, w)\right) \gamma(z)$ является строго убывающей $\quad$ и $\mu \tau f\left(l^{*}(\bar{z}, w)\right) \gamma(\bar{z})=0$. Так как мы рассматриваем задачу на максимизацию, $\lambda \geq 0$. Из-за того что функция $\mu \tau f\left(l^{*}(z, w)\right) \gamma(z)$ непрерывна и монотонна, будет существовать не более одного значения $\tilde{z} \in\left[z^{*}, \bar{z}\right]$ такого, что $\mu \tau f\left(l^{*}(\tilde{z}, w)\right) \gamma(\tilde{z})-\lambda c=0$.

Предположим, что не существует такого $\tilde{z}$. Тогда $\forall z \in\left[z^{*}, \bar{z}\right]$ $\mu \tau f\left(l^{*}(z, w)\right) \gamma(z)-\lambda c<0$ (случай с обратным знаком невозможен, потому что $\lambda c \geqslant 0$ и $\left.\mu \tau f\left(l^{*}(\bar{z}, w)\right) \gamma(\bar{z})=0\right)$. Тогда функция Лагранжа будет убывать по $p(z)$ и налоговый инспектор выберет $p=0$ для всех $z$. В таком случае бюджетное ограничение аудитора будет выполняться как строгое неравенство, и, в соответствии с условием дополняющей нежесткости, множитель Лагранжа $\lambda=0$. Тогда $\bar{z}$ является решением $\mu \tau f\left(l^{*}(z, w)\right) \gamma(z)-\lambda c=0$, что противоречит нашему изначальному предположению.

Найдем теперь это значение $\tilde{z}$. Для $z<\tilde{z}$ функция Лагранжа возрастает по $p$, так что аудитор выберет максимальное значение $p(z)=1 / \mu$. Для $z>\tilde{z}$ лагранжиан убывает по $p$, что даст $p=0$ для этих значений $z$. Заметим, что $p(z)$ не возрастает по $z$, так что достаточное условие для ограничения совместимости по стимулам выполняется.

Покажем, что $\tilde{z}$ является решением следующего уравнения: 


$$
\int_{z}^{z} z c \frac{1}{\mu} g(z) d z=C .
$$

Для начала мы покажем, что эта догадка весьма обоснована. Рассмотрим бюджетное ограничение (выполняющееся как равенство)

$$
\begin{aligned}
& C=\int_{z}^{\bar{z}}{ }^{*} c p(z) g(z) d z=\int_{z}^{\tilde{z}} * c p(z) g(z) d z+\int_{\tilde{z}}^{\bar{z}} c p(z) g(z) d z= \\
& =\int_{z}^{\tilde{z}} * c \frac{1}{\mu} g(z) d z+\int_{\tilde{z}}^{\bar{z}} c \cdot 0 \cdot g(z) d z,
\end{aligned}
$$

что совпадает с (15).

Во-вторых, предположим противное: $\hat{z} \neq \tilde{z}$ является решением (15). Невозможно, чтобы $\tilde{z}>\hat{z}$, потому что в этом случае бюджетное ограничение нарушается. Если $\tilde{z}<\hat{z}$, тогда бюджетное ограничение выполняется как строгое неравенство, что дает возможность аудитору возможность проверить еще одного агента с вероятностью $1 / \mu$ и увеличить значение целевой функции, не нарушив этим бюджетное ограничение. Таким образом, $\tilde{z}$ на самом деле является решением (15)

Полученный результат является общим для таких моделей аудита и соответствует результатам, полученными ранее $[7,6,15]$. В [15] предлагается строгое математическое доказательство для этого решения в виде «релейного переключения».

Решение задачи аудитора. Поиск $z^{*}$. Найдем теперь оптимальный уровень отсечения $z^{*}$. Для этого мы продифференцируем функцию Лагранжа по $z^{*}$ :

$$
\begin{aligned}
& \partial \mathfrak{L} / \partial z^{*}=f\left(l^{*}\left(z^{*}, w\right)\right)\left(1-\int_{z^{*}}^{\bar{z}} l^{*}(z, w) g(z) d z\right)+\left(\pi\left(z^{*}, w\right)-w\right) l^{*}\left(z^{*}, w\right) g\left(z^{*}\right)- \\
& -p\left(z^{*}\right)\left[\mu \tau f\left(l^{*}\left(z^{*}, w\right)\right) \gamma\left(z^{*}\right)-\lambda c\right] g\left(z^{*}\right) .
\end{aligned}
$$

Заменив $p\left(z^{*}\right)$ на оптимальное значение $p$ в этой точке и использовав условие равновесия на рынке труда (13) и отношение Миллса, мы получим

$$
\begin{aligned}
& \frac{\partial \mathfrak{L}}{\partial z^{*}}=f\left(l^{*}\left(z^{*}, w\right)\right)\left(1-G\left(z^{*}\right)\right)+\left(\pi\left(z^{*}, w\right)-w\right) l^{*}\left(z^{*}, w\right) g\left(z^{*}\right)- \\
& -\tau f\left(l^{*}\left(z^{*}, w\right)\right)\left(1-G\left(z^{*}\right)\right)+\frac{\lambda c}{\mu} g\left(z^{*}\right)=(1-\tau) f\left(l^{*}\left(z^{*}, w\right)\right)\left(1-G\left(z^{*}\right)\right)+ \\
& +\frac{\lambda c}{\mu} g\left(z^{*}\right)+\left(\pi\left(z^{*}, w\right)-w\right) l^{*}\left(z^{*}, w\right) g\left(z^{*}\right) .
\end{aligned}
$$

Так как $\lambda>0$ и $\forall z>z_{0} \pi(z, w)>w$, получаем, что $\partial \mathfrak{L} / \partial z^{*}>0$. Это значит, что аудитор выберет наибольшее значение $z^{*}$ из всех возможных и это $z_{\tau}$. 
Этот результат логичен и интуитивно понятен. Если бы налоговый инспектор выбрал $z^{*}<z_{\tau}$, тогда, учитывая то, что вероятность аудита равна $1 / \mu$ для группы предпринимателей с низким уровнем $z$, этим предпринимателям пришлось бы покинуть рынок и налоговой инспекции пришлось бы тратить средства на их аудит впустую. С другой стороны, если бы аудитор хотел, чтобы эти агенты остались среди производителей, ему пришлось бы снизить вероятность их аудита, и это либо было бы несовместимо с условием неубывания $p(z)$, либо заставило аудитора снизить вероятность проверок агентов с $z>z_{\tau}$, что не является оптимальным выбором. С качественной точки зрения тот факт, что $z^{*}=z_{\tau}$, интуитивно означает, что налоговая инспекция будет нетерпимой к агентам, которые не смогут (даже при всем их желании) заплатить налоги полностью.

Решение задачи аудитора. Поиск $\boldsymbol{x}(\boldsymbol{z})$. Так как $p(z)=1 / \mu$ для $z \in$ $\in\left[z_{\tau}, \tilde{z}\right)$, эти агенты будут декларировать свои доходы полностью: $x(z)=$ $=\pi(z, w)$. Мы предположим, что фирма $\tilde{z}$ также декларирует всю свою прибыль. Агенты с $z>\tilde{z}$ не проверяются, поэтому они выберут наименьшее значение декларируемой прибыли $x$ среди доступных для них.

Рассмотрим агента $\hat{z}>\tilde{z}$. В соответствии с (6) и оптимальным выбором $p(z)$

$$
\begin{aligned}
& U(\hat{z}, w)=U(\tilde{z}, w)+\int_{\tilde{z}}^{\hat{z}} \mu p(z) \tau f\left(l^{*}(z, w)\right) d z=U(\tilde{z}, w), \\
& U(\tilde{z}, w)=x(\tilde{z}) \tau+\mu p(\tilde{z}) \tau(\pi(\tilde{z}, w)-x(\tilde{z}))=x(\tilde{z}) \tau=U(\hat{z}, w)=x(\hat{z}) \tau .
\end{aligned}
$$

Это значит, что агенты с типами $z>\tilde{z}$ будут декларировать в качестве своей прибыли прибыль фирмы $\tilde{z}$. Результат убедительный: фирмы, которые никто не проверяет, декларируют минимальный размер прибыли, при котором налоговый инспектор не станет проверять эти фирмы. Если бы фирма решила декларировать еще более низкий уровень прибыли, тогда аудитор (который на самом деле не знает $z$ и строит свои стратегии как $p(x)$, а не $p(z))$ подумал бы, что у фирмы низкий уровень $z$ и проверил эту фирму с вероятностью $1 / \mu$, что крайне нежелательно для фирмы.

4.6. Оптимальный механизм аудита. Перечислим полученные результаты:

$$
z^{*}=z_{\tau}, \quad p(x)=\left\{\begin{array}{ll}
1 / \mu, & \text { если } x<\pi(\tilde{z}, w) ; \\
0, & \text { если } x \geqslant \pi(\tilde{z}, w),
\end{array} \quad x(p)= \begin{cases}\pi(z, w), & \text { если } p \geq 1 / \mu \\
\pi(\tilde{z}, w), & \text { если } p<1 / \mu,\end{cases}\right.
$$

где $\tilde{z}$ является решением (15). В рассматриваемом случае с постоянными 
предельными издержками аудита (15) упрощается до $\mu \frac{C}{c}=G(\tilde{z})--G\left(z_{\tau}\right)$.

В равновесии сумма доходов государственного бюджета (значение целевой функции аудитора) составит

$$
\begin{aligned}
& T=\int_{z}^{\bar{z}}{ }^{*}[x(z) \tau+\mu p(z) \tau(\pi(z, w)-x(z))] g(z) d z= \\
& =\int_{z_{\tau}}^{\tilde{z}} \tau \pi(z, w) g(z) d z+\tau \pi(\tilde{z}, w)(1-G(\tilde{z})) .
\end{aligned}
$$

Заметим, что в равновесии сумма штрафов равна нулю, так как те агенты, которых аудитор проверяет, платят налоги полностью, а нарушающих (причем не очень сильно) законодательство агентов аудитор не проверяет.

Это вполне соответствует действительности: по данным бюджетной статистики, сумма штрафов и пени достаточно низка по сравнению с суммой собранных налогов, но, тем не менее, фирмы боятся налоговых проверок. Тем не менее штрафы оказывают важное влияние с теоретико-игровой точки зрения: они играют роль правдоподобной угрозы, которая пугает фирмы и заставляет их либо платить все налоги, либо платить хотя бы их бо́льшую часть.

Проанализируем зависимость налоговых поступлений от «штрафного параметра» $\mu>1^{7}$. Можно найти производную значения целевой функции по $\mu$

$$
\frac{\partial T}{\partial \mu}=\tau \gamma(\tilde{z}) \frac{C}{c} f\left(l^{*}(\tilde{z}, w)\right)>0 .
$$

Это достаточно очевидный результат: чем выше штрафы, тем больше агентов будут платить налоги полностью. Кроме того, видно, что эта производная убывает по $\tilde{z}$ в силу выполнения условия (14). В свою очередь, (15) дает нам понять, что $\tilde{z}$ возрастает по $\mu$, что значит, что $T$ - вогнутая функция по $\mu$. Кроме того, очевидно, что при прочих равных увеличение отношения $C / \mathrm{c}$ (увеличение, не нарушающее ограничение на максимальное $C$ ) приведет к увеличению как скорости возрастания функции $T(\mu)$, так и степени ее вогнутости.

${ }^{7}$ Случай $\mu=1$ является тривиальным. В этом случае все фирмы будут «забывать» платить налоги, а при налоговой проверке предприниматели будут возвращать неоплаченную сумму в казну. С математической точки зрения все наши результаты имеют смысл лишь при $\mu>1$. 
Если $\mu$ достаточно велико, то будет нарушаться ограничение, которое мы по смыслу ввели на $C$, однако с математической точки зрения задача будет иметь решение, хотя и тривиальное. Все будут платить налоги, и мы приблизимся к ситуации с симметричной информацией. Похожим образом на значение целевой функции влияет и бюджет налоговой инспекции:

$$
\frac{\partial T}{\partial C}=\lambda=\frac{\mu}{c} \tau f\left(l^{*}(\tilde{z}, w)\right) \gamma(\tilde{z}) .
$$

Эта производная также положительна и убывает по $C$. Наибольший интерес вызывает сравнение этой производной с единицей. В случае если производная больше единицы, то небольшое увеличение бюджета налоговой инспекции выгодно правительству, так как приведет к росту налоговых сборов на бОльшую величину по сравнению с увеличением издержек на аудит. В общем случае сравнить значение этой производной с единицей - задача непростая, поэтому мы сделаем это в рамках численного анализа равновесия в модели для заданных функций $g(z), f(l)$.

При больших значениях $C$ задача также вырождается в тривиальную, и сумма налоговых сборов становится равной налоговым поступлениям при наблюдаемом $z$.

\section{5. Численный анализ равновесия в модели}

В рамках численного анализа равновесия в модели с асимметрией информации мы предположим степенную производственную функцию $f(l)=$ $=l^{\alpha}$ и равномерное распределение $z \sim U[1,2]$, как это было сделано в работе [13]. Заметим, что сочетание равномерного распределения параметра $z$ и степенной производственной функции удовлетворяет условию (14). В таком случае условие (15) еще сильнее упростится до линейного $\tilde{z}=\mu \frac{C}{c}+z_{\tau}$.

Требование, которое вводится на $C$, чтобы решение не было тривиальным, сводится к

$$
\mu \frac{C}{c}<2-z_{\tau} .
$$

На рис. 2 изображены кривые Лаффера для $\alpha=1 / 2$ и $1 / 3$ для случаев, когда информация о типах агентов симметрична (пунктирные линии) и когда она ненаблюдаема (сплошные линии). В качестве параметра $\mu$ мы взяли 1,2, что значит, что нарушители налогового законодательства должны вернуть за- 
долженность по налогам и заплатить 20\% штрафа. Параметры $C$ и $c$ выбраны так, чтобы их отношение равнялось 0.05 , что означает, что аудитор мог бы проверить 5\% фирм с вероятностью, равной единице ${ }^{8}$.

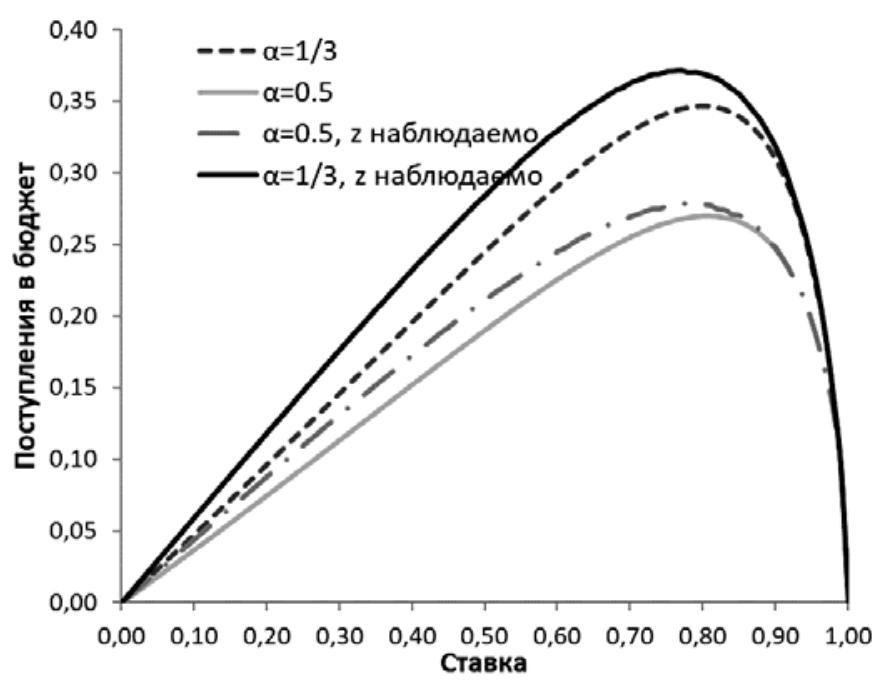

Рис.2. Зависимость налоговых поступлений от $\tau$ для симметричной и асимметричной информации.

Заметно, что целые линии заканчиваются при $\tau<1$. Это связано именно с требованием (17): при слишком высоких $\tau$ оно не выполняется. Однако мы видим, что при приближении к правой границе области своего определения функция налоговых поступлений при асимметричной информации сходится к функции налоговых поступлений в случае наблюдаемого $z$, о чем мы говорили в конце предыдущего раздела.

Для меньших значений $\tau$ мы видим, что сумма налоговых сборов в случае, когда информация симметрична, выше, по сравнению с ситуацией асимметрии информации, ибо в первой никто не уклоняется от налогов. Кроме того, немного меняются (увеличиваются) точки максимума кривых Лаффера для обоих значений $\alpha$ по сравнению с ситуацией, когда информация симметрична.

Рис.3 иллюстрирует наши выводы относительно зависимости суммы налоговых поступлений от «штрафного» параметра $\mu$ для двух различных отношений $C / c$ и для $\alpha=0.5, \tau=0.2$. На графике видно, что функция налоговых доходов от $\mu$ является возрастающей и вогнутой, причем при достаточно больших значениях $\mu$, при которых неравенство (17) начинает нарушаться, значение функции становится равным сумме налоговых сборов, полученных государством при симметричной информации.

\footnotetext{
${ }^{8}$ Хотя он этого не будет делать, так как это не оптимальная стратегия.
} 
Модель общего равновесия с налоговым аудитом и эндогенным выбором ...

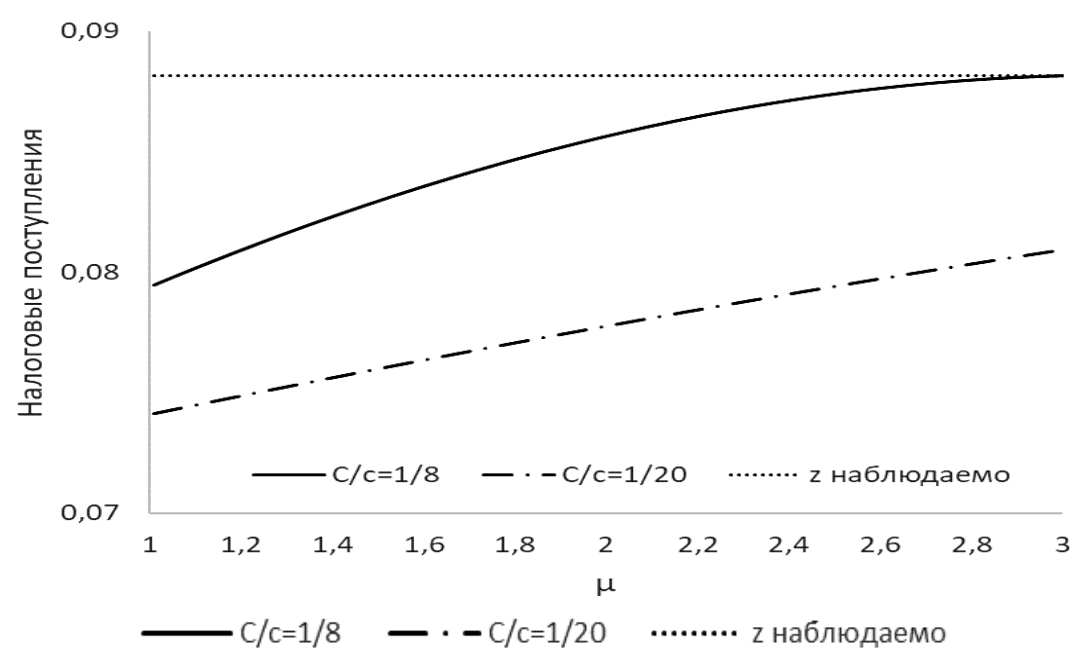

Рис.3. Зависимость налоговых поступлений от $\mu$.

График подтверждает наши выводы относительно того, что относительное увеличение ресурсов аудитора приведет к росту налоговых поступлений, равно как и к росту первой и второй производных функции $T(\mu)$.

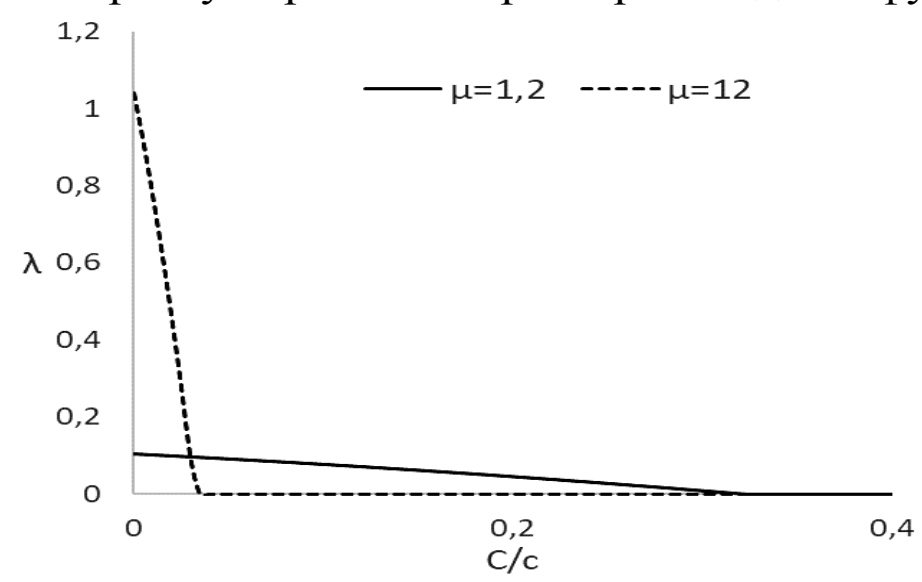

Рис.4. Зависимость $\lambda$ от $C / c$.

На рис.4 изображена зависимость двойственной переменной $\lambda$ от отношения $C / c$. Для разумных уровней $\mu$ (на графике изображен вариант с $\mu=1,2), \lambda$ сильно меньше единицы, что значит, что с каждого дополнительного рубля, потраченного на аудит, государство соберет меньше одного рубля налогов (при $\tau=0,2$ и при $C / c=1$ ). Для неоправданно больших уровней $\mu$ (нижняя граница находится вблизи $\mu=12$, этот случай и изображен на графике) и для малых значений $C / c$ и $\lambda$ принимает значения, большие или равные единице. Это означает, что государству выгодно увеличивать финансирование аудитора до уровня 1/1000. Таким образом, мы смогли удостовериться, что при определенном наборе параметров (не очень реалистичном) увеличивать финансирование налоговой инспекции может быть выгодно. 


\section{6. Анализ полученных результатов и дальнейших направлений работы}

В настоящей работе рассматривается модель общего равновесия, описывающая взаимодействие неоднородных агентов, выбирающих между рынком труда и самозанятостью, и государством, играющим в модели роль проверяющего органа (аудитора), контролирующего факт ухода от налогов. Полученные в указанных условиях результаты не противоречат результатам, полученным в моделях, в которых отсутствует рынок труда, например, [6,7].

Мы показали, что кривые Лаффера в случае асимметрии информации изменяются по сравнению с ситуацией, когда фирмы налоги платят полностью $[13,14]$. В частности, увеличивается ставка налогообложения, максимизирующая сумму налоговых поступлений. Кривая Лаффера при асимметрии информации приближается к своему аналогу при симметричной информации, если высоки штрафы или бюджет налоговой инспекции. Несмотря на то, что в равновесии сумма выплачиваемых штрафов равна нулю, они оказывают существенное влияние на сумму налоговых поступлений. Являясь эффективным методом устрашения нарушителей налогового законодательства, они увеличивают сумму налоговых сборов с замедляющимся темпом. Таково же влияние бюджета налоговой инспекции.

Полученные результаты предполагают ряд дальнейших исследований. Во-первых, развитие задачи аудитора за счет введения дополнительных ограничений на используемую им информацию (в частности, предположение, что аудитор может различать работников и самозанятых) и снятие предпосылок, избыточно упрощающих анализ. Во-вторых, крайне перспективным представляется исследование моделей общего равновесия с неоднородными агентами и анализ государственной налоговой политики.

\section{СПИСОК ЛИТЕРАТУРЫ}

1. R. Gordon, W. Li. Tax structures in developing countries: Many puzzles and a possible explanation // Journal of Public Economics, 2009, 93, p.855-866.

2. T. Besley, T. Persson. The origins of state capacity: property rights, taxation, and politics // NBER WORKING PAPER SERIES, 2007.

3. M. Keen. VAT, Tariffs, and Withholding: Border Taxes and Informality in Developing Countries // IMF Working Paper, 2007, July.

4. A. Yakovlev. Black cash tax evasion in Russia: its forms, incentives and consequences at firm level // Europe-Asia Studies, 2001, 52 (1).

5. P. Pestieau, U.M. Possen. Tax evasion and occupational choice // Journal of Public Economics, 1991, v.45, №1, p.107-125.

6. I. Sanchez, J. Sobel. Hierarchical design and enforcement of income tax policies // Journal of Public Economics, 1993, 50, p.345-369. 
7. S. Bigio, E. Zilberman. Optimal self-employment income tax enforcement // Journal of Public Economics, 2011, 95, p.1021-1035.

8. R. Lucas. On the Size Distribution of Business Firms. The Bell Journal of Economics // 1978, 9 (2), p.508-523.

9. B. Jovanovic. Firm Formation with Heterogeneous Management and Labor Skills // Small Business Economics, 1994, №6, p.185-191.

10. Д.А. Покровский. Способность к предпринимательству: структура занятости и неравенство доходов // Пространственная экономика, 2014, №2, с.9-39;

D.A. Pokrovskii. Sposobnost k predprinimatelstvu: struktura zaniatosti i neravenstvo dokhodov // Prostranstvennaia ekonomika, 2014, №2, s.9-39.

11. Д.А. Покровский, А.Б. Шаповал. Распределение предпринимательских способностей и миграция: структура занятости, неравенство доходов и благосостояние // Журнал Новой экономической ассоциации, 2015, №2, с.36-62;

D.A. Pokrovskii, A.B. Shapoval. Raspredelenie predprinimatelskikh sposobnostei i migratsiia: struktura zaniatosti, neravenstvo dokhodov i blagosostoianie // Zhurnal Novoi ekonomicheskoi assotsiatsii, 2015, №2, s.36-62.

12. В.А. Шарунова, А.В. Аистов, С.И. Кичко, Д.А. Покровский. Распределение предпринимательских способностей и производительности: структура рынка труда // Экономический журнал ВШЭ, 2015, т.19, №2, с.218-248;

V.A. Sharunova, A.V. Aistov, S.I. Kichko, D.A. Pokrovskii. Raspredelenie predprinimatelskikh sposobnostei i proizvoditelnosti: struktura rynka truda // Ekonomicheskii zhurnal VShE, 2015, t.19, №2, s.218-248.

13. Ю.К. Ачкасов, Н.П. Пильник. Влияние налога на прибыль на экономические показатели в условиях эндогенного выбора между рынком труда и предпринимательством // Журнал Новой экономической ассоциации, 2017, №1(33), с.12-27;

Iu.K. Achkasov, N.P. Pilnik. Vliianie naloga na pribyl na ekonomicheskie pokazateli v usloviiakh endogennogo vybora mezhdu rynkom truda i predprinimatelstvom // Zhurnal Novoi ekonomicheskoi assotsiatsii, 2017, №1(33), s.12-27.

14. Ю.К. Ачкасов, Н.П. Пильник. Оптимальный механизм аудита налога на прибыль и эндогенный выбор между рынком труда и самозанятостью // В кн.: XVI Апрельская международная научная конференция по проблемам развития экономики и общества: в 4 кн. / Отв. ред.: Е.Г. Ясин. Кн.1. - М.: Изд. дом НИУ ВШЭ, 2016, с.467-476; Iu.K. Achkasov, N.P. Pilnik. Optimalnyi mekhanizm audita naloga na pribyl i endogennyi vybor mezhdu rynkom truda i samozaniatostiu // V kn.: XVI Aprelskaia mezhdunarodnaia nauchnaia konferentsiia po problemam razvitiia ekonomiki i obshchestva: v 4 kn. / Otv. red.: E.G. Iasin. Kn.1. - M.: Izd. dom NIU VShE, 2016, s.467-476.

15. S. Morton. Strategic Auditing for Fraud // The Accounting Review, 1993, 68 (4), p.825-839.

16. R.B. Myerson. Optimal Auction Design // Mathematics of Operations Research, 1981, 6 (1), p.58-73.

17. W. Samuelson. Bargaining under Asymmetric Information // Econometrica, 1984, 52 (4), p.995-1006. 